\title{
The Running Practice Modulates Inflammatory Markers and not Alters Global DNA Methylation in Elderly Men
}

\author{
João José Cunha1 ${ }^{1}$ Anelise Ineu Figueiredo ${ }^{1}$, Gilson Pires Dorneles ${ }^{2}$, Alessandra Peres ${ }^{2}$, Daniela \\ Pochmann", Viviane Rostirola Elsner ${ }^{1,}$, Maristela Padilha de Souza $^{1}$. \\ ${ }^{1}$ Programa de Pós-Graduação em Biociências e Reabilitação do Centro Universitário Metodista do IPA, Porto Alegre, Rio \\ Grande do Sul, Brazil. \\ ${ }^{2}$ Programa de Pós-Graduação em Ciências da Reabilitação, Universidade Federal de Ciências da Saúde de Porto Alegre, \\ Porto Alegre, Rio Grande do Sul, Brazil.
}

Received: December 01, 2017; Accepted: December 12, 2017; Published: December 23, 2017

*Corresponding author: Viviane R. Elsner, PhD, Institution: Centro Universitário Metodista IPA, Rua Coronel Joaquim Pedro Salgado, 80-Rio Branco, Porto Alegre, Rio Grande do Sul CEP 90420-060, Brazil, Tel: 55(51)33161200;Email: elsner.viviane@gmail.com

\begin{abstract}
Objectives: The current study aimed to evaluate 1) the impact of the regular running practice 2) as well the acute effect of running race on inflammatory markers and DNA methylation status in peripheral blood of health elderly men.

Methods: Fifteen male volunteers aged 60 years and older were recruited. They were allocated in two groups: runners (RUN, $n=8)$ and sedentary (SED, $n=7$ ), considering if they were sedentary or amateur street runners. All participants, SED and RUN groups, were submitted to a basal blood collection. After 5 days, two blood samples were collected in the RUN group: 30 min before and immediately after a $5-10 \mathrm{~km}$ outdoor running performance.The blood samples $(15 \mathrm{~mL})$ were collected for the epigenetic and inflammatory measurements, which were done using specific kits, according to the manufacturer's instructions.
\end{abstract}

Results: FIn the basal period, no significant differences were observed on DNA global methylation status between the SED and RUN groups. However, the SED group showed increased IL- 4 and IL- 6 levels when compared to the RUN individuals $(p=0,040$ and 0,035 , respectively). The IL-6 and IL-4 levels were significantly higher in the EXE group after the running performance compared to the before running period ( $\mathrm{p}=0,004$ and 0,013 respectively), while DNA methylation status remained unaltered.

Conclusion: Our data suggest that DNA methylation status might not be consider a potential biomarker related to the healthy elderly runners phenotype. Furthermore, the inflammatory response may indicate a possible regulatory effect provided by the endurance exercise in the elderly group because IL- 4 and IL- 6 are synergic cytokines directing an immune response.

Keywords: Exercise; Running; Aging; Epigenetic; Inflammation;

\section{Introduction}

Aging has become increasingly in the world population and the projection for 2050 is that people over 60 years old be around 2 billion [1]. To clarify specific mechanisms of the biological aging process and the elaboration of combined strategies for the management of elderly health are urgent in order to guarantee the quality of life of this population. Emerging experimental and clinical evidences suggest that the imbalance of epigenetic machinery plays a pivotal role in both normal aging process as well in age-related diseases [2,3]. Epigenetic process result in heritable phenotypical alterations in the expression of specific genes without alter the primary DNA sequence. The hallmarks of epigenetic gene regulation include DNA methylation and histone acetylation levels modifications [4].

DNA methylation is a process catalyzed by DNA methyl transferees (DNMTs) enzymes and is usually associated with transcriptional repression and gene silencing [5]. Histone acetylation status is regulated by histone acetyl transferees (HATs) and histone deacetylases (HDACs). HATs produce a larger chromatin structure, characteristic that allows transcriptional activation, whereas HDACs exert the opposite effect [6].

Nowadays, it is observed increased research on both pharmacological and non-pharmacological tools to prevent and minimize the physiological age modifications and diseases. In this context, exercise has been pointed out as an interesting strategy promoting several benefits in the elderly individuals [7]. In this context, a recent study conducted by Zhang and colleagues (2015) showed that DNA methylation levels are modulated in elderly people previously sedentary submitted to an interval walking training protocol. Furthermore, this response was related to an improvement on inflammatory status, suggesting that exercise might induce beneficial effects on health by engaging epigenetic signals [8].

Among the modalities of physical exercise, recreational running is markedly growing as an option, even among the aged population. The beneficial impact of running practice on human physiological systems it is well described [9]. It was recently reported the impact of the running practice on epigenetic modulation in middle-aged individuals [10]. However, this response in elderly individuals has been poorly investigated. Furthermore, although many studies have shown that exercise can induce epigenetic changes in peripheral blood of different populations $[11,12]$ it is important to note that most 
of these investigations are conducted with previously sedentary individuals, while findings with well-trained populations are scarce.

Importantly, age-related changes of immune system contribute to increased ratios of infection diseases, poor vaccine response and cardio metabolic diseases which are linked with the chronic low grade inflammation observed in elderly subjects [13]. This process is namely immuno senescence and was mediated by change in both cellular adaptive and innate immune system, as well as in pro and anti-inflammatory cytokine levels such as interleukin-1 $\beta$ (IL-1 $\beta$ ), IL-6, and Tumour Necrosis Factor-Alpha $(\mathrm{TNF}-\alpha)[14]$. Cytokines produced by immune cells play a critical role in the development of host immunity against infection. It has been established that Interferon-gamma (INF- $\gamma$ ) represents a type 1 (T1) cytokines, which are involved in cellular immune response $[15,16]$. In contrast, interleukin-4 (IL-4) participate of type 2 (T2) immune response against extra-cellular/humoral pathogens. In addition, $\mathrm{T} 1$ and $\mathrm{T} 2$ cytokines responses are mutually inhibitory [17]. Thus, the INF- $\gamma /$ IL-4 ratio has been used as an indicator of immune polarization [18]. Currently, it is well established that an acute bout of strenuous endurance exercise inhibits the responses of T1 cytokines due to actions of glucorticoids and changes in intracellular transcription factors in immune cells $[19,20]$. In addition, these changes can contribute to anti-inflammatory effects of exercise in active exercisers.

Finally, it is known that long-term endurance exercise training also modulates inflammatory markers, specifically, is able to induce a reduction in serum interleukin 6 (IL-6) and interleukin 4 (IL-4) levels [21]. In addition, cardio respiratory fitness is inversely associated with several markers of inflammation, such as IL-6, demonstrating that exercise training might plays a pivotal key for the control of low-grade inflammation [22]. Data from epidemiological studies demonstrated an association between the levels of physical activity, lower levels of bacterial and viral infections, as well as distinct immune profile in elderly subjects characterized by low levels of pro inflammatory mediators $[23,24]$. Nevertheless, to date, no study evaluated the impact of acute outdoor endurance training on cytokine markers in welltrained elderly individuals.

In view of these considerations, the aim of the present study was to compare the basal levels of inflammatory parameters (IL4 and IL-6) and global DNA methylation status in the peripheral blood from elderly healthy amateur runners (RUN group) with elderly sedentary individuals (SED group). In order to investigate the acute outcomes of exercise in trained individuals, we also analyzed the effect of a running race $(5-10 \mathrm{~km})$ on these markers in the EXE group.

\section{Material and Methods}

\section{Ethical Procedures}

The study was approved by the Ethics Committee of Methodist University Center IPA (protocol number 1190948) and all participants provided informed written consent prior to participation.

\section{Subjects}

Participants off both groups (RUN and SED) were recruited through an advertisement on the Social Network Sites. They were allocated in two groups: runners (RUN, $n=8)$ and sedentary (SED, $n=7$ ), taking into account the question that they were sedentary or amateur street runners. As criteria to compose RUN group, volunteers should be regular amateurs runners, practicing running bouts three times a week for at least two years. The amount of physical activity in the RUN group was accessed by a self-report questionnaire of training characteristics. To be included in SED group, individuals should not have engaged in regular practice of physical exercise in the last six months prior to data collection.

Exclusion criteria for both groups were the presence of chronic diseases, the use of drug-containing HDAC inhibitors and smoking. The participants were oriented not to use antiinflammatory and antibiotic medicines $72 \mathrm{~h}$ before all blood collections. All volunteers should be physically independent and do not have cognitive limitations that could prevent the understanding of tests.

\section{Experimental Trials}

In this comparative, controlled, cross sectional study, volunteers went to the Laboratory of Exercise Physiology at the Centro Universitário Metodista-IPA after a twelve-hour of fasting period and blood samples $(15 \mathrm{ml})$ were obtained from an antecubital vein (basal period) for measurement of inflammatory and epigenetic measurements. The body composition and the body mass index (BMI) in both group were also assessed.

\section{Global DNA Methylation Levels Analysis}

Global DNA methylation levels in the plasma were quantified using Methyl Flash TM Methylated DNA Quantification Kit (Epigentek, Catalog number P-1034, NY, USA) according to the manufacturer's information. Capture antibody in this kit binds to 5-methylcytosine and it was measured the total DNA methylation level as a percentage of total DNA present in each plasma sample. A standard curve with positive and negative controls was run with the samples. Thus, $100 \mathrm{ng}$ of DNA $(1-8 \mathrm{ng} / \mu \mathrm{l})$ was bound to the plate at $370 \mathrm{C}$ for $90 \mathrm{~min}$. The methylated fraction of DNA was detected using capture and detection antibodies. The relative optical density (OD) units were quantified by reading the absorbance in a FLUOstar Optima micro plate reader (Labtech). Methylated DNA amount was proportional to the OD measured. Absolute amount of methylated DNA was quantified from a standard curve, and the slope of the standard curve was used to calculate the percentage of methylated DNA $(5-\mathrm{mC} \%)$ in the plasma samples.

\section{Cytokines Measurement}

The levels of IL- 4 and IL- 6 in the plasma were determined by Enzyme-Linked Immunos or bent Assay (ELISA) protocols according the manufacturer's recommendations. IL-4 and IL-6 were analyzed using ELISA Development Kit of Peprotech Inc, New Jersey, USA. 


\section{Statistical analysis}

Data normality was verified by the Shapiro-Wilk test. To assess the differences in normally distributed and nonparametric variables, the $t$ test and the Mann-Whitney test were used. Characteristics of volunteers was presented as media \pm standard derivation or absolute frequency (\%). To compare anthropometric parameters, DNA methylation levels and inflammatory markers between the RUN and SED groups, we utilized an unpaired t test. Pearson correlation was used to identify the evaluated correlations. PASW Statistics 20.0 for Windows (SPSS) was used to perform Statistical analyses. The significance level was set to $5 \%(\mathrm{p}<0,05)$.

\section{Results}

Participants characteristics (SED and RUN groups) are highlighted in Table 1 \& Table 2 illustrates the training characteristics of the RUN group.

In the basal period, DNA methylation levels did not differ between groups, as highlighted in Figure 1. Regarding the inflammatory markers, the SED group showed increased values of IL-4 ( $p=0.040)$ and IL-6 ( $p=0.035)$ when compared to RUN group (Figure 2).

In the race day, the RUN group was encouraged to exercise to their limit. The DNA methylation levels remained unaltered comparing the before and after the outdoor running performance ( $p=0.081$, Figure 3). However, as showed in Figure 4, higher IL-6 levels were observed after the running compared to the before period $(\mathrm{p}<0.05)$.

Table 1: General characteristics of the volunteers

\begin{tabular}{|c|c|c|}
\hline Variables & SED (n=7) & RUN (n=8) \\
\hline Age (years) & $64.8 \pm 4.2$ & $64.2 \pm 3.9$ \\
\hline Weight (Kg) & $73.1 \pm 5.78$ & $71.1 \pm 5.79$ \\
\hline Height (cm) & $172 \pm 0.03$ & $174 \pm 0.04$ \\
\hline BMI $(\mathrm{kg} / \mathrm{m} 2)$ & $24.5 \pm 2.30$ & $23.3 \pm 1.34$ \\
\hline
\end{tabular}

Table 2: Data related to training characteristics of the Running group $(n=8)$

\begin{tabular}{|c|c|c|}
\hline \multicolumn{2}{|c|}{ Variables } & Frequency \\
\hline \multirow{2}{*}{ Running Experience (years) } & 4 -Feb & $50 \%$ \\
\cline { 2 - 3 } & 20 -Oct & $25 \%$ \\
\cline { 2 - 3 } & $>20$ & $25 \%$ \\
\hline \multirow{2}{*}{ Frequency (sessions/week) } & 3 -Feb & $75 \%$ \\
\hline \multirow{2}{*}{ Session duration (minutes) } & $6-\mathrm{Apr}$ & $25 \%$ \\
\hline & 60 & $75 \%$ \\
\hline
\end{tabular}

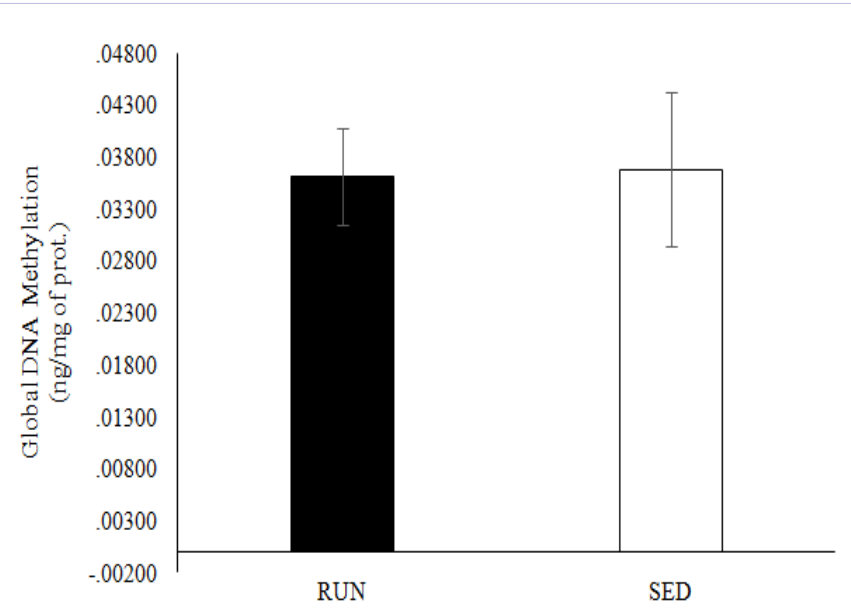

Figure 1: Basal global DNA methylation levels of running (RUN) and sedentary (SED) groups. Values are expressed as mean \pm SD

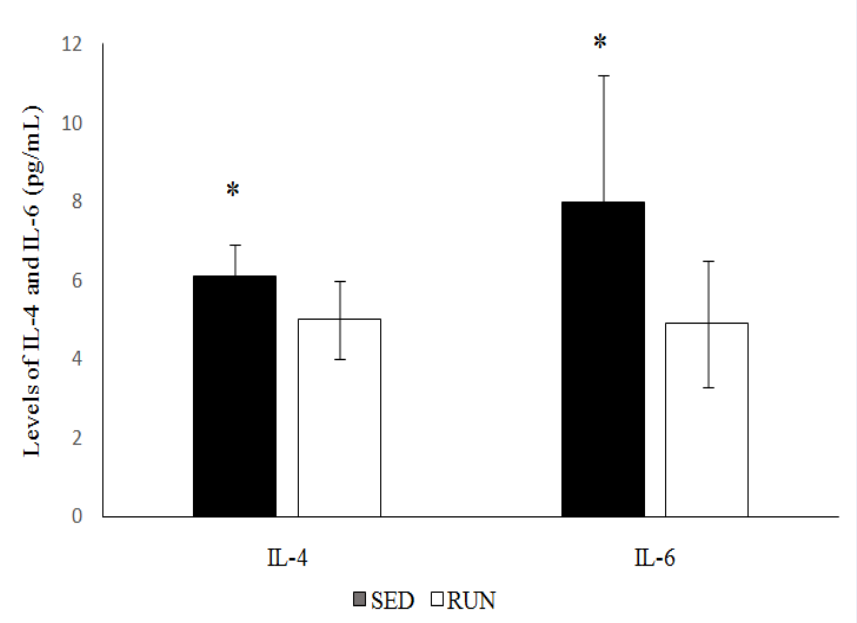

Figure 2: Basal plasmatic levels of IL-4 and IL-6 of sedentary (SED) and running (RUN) groups. Values are expressed as mean \pm SD.* Denotes statistical difference when compared with pre period $(\mathrm{p}<0.05)$

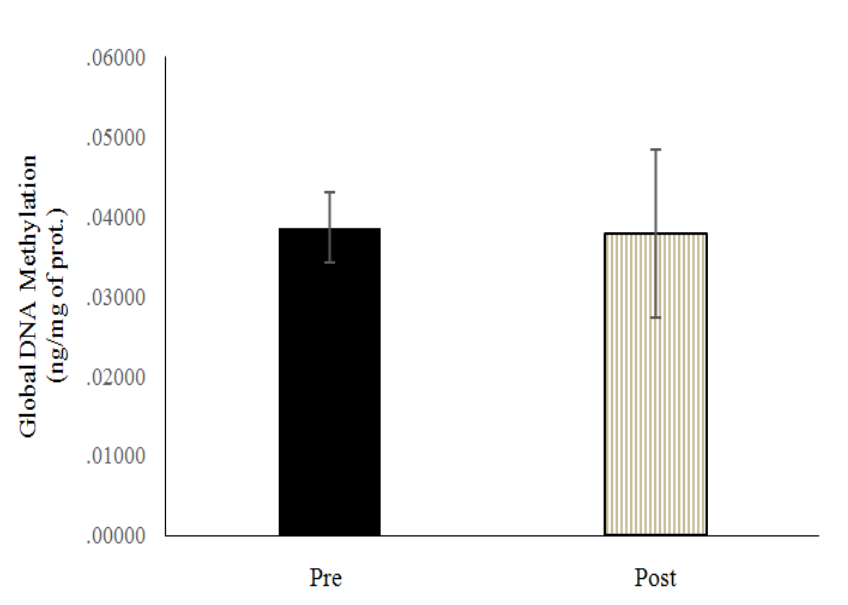

Figure 3: Global DNA methylation of running group (RUN) in response to acute exercise performance. Values are expressed as mean \pm SD. 


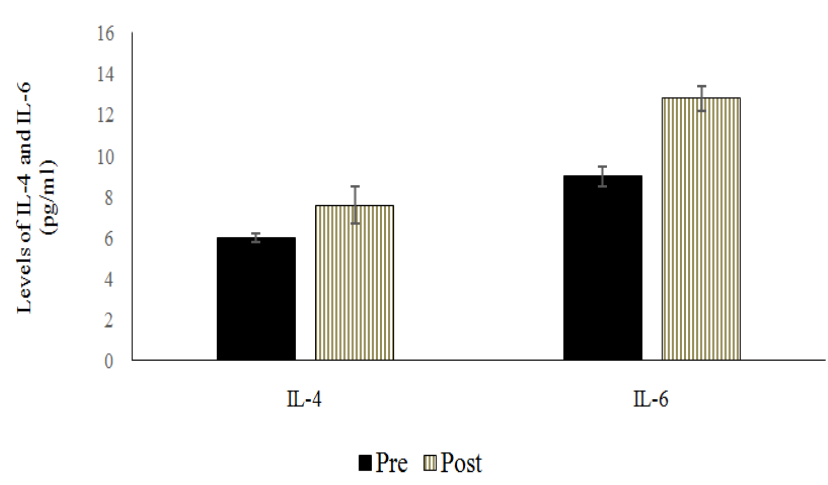

Figure 4: Plasmatic levels of IL-4 and IL-6 of running group (RUN) in response to acute physical exercise performance. Values are expressed as mean \pm SD.* Denotes statistical difference when compared with pre period $(\mathrm{p}<0.05)$.

\section{Discussion}

To our knowledge, this is the first study that investigated the effects of acute and chronic physical exercise exposure, focused on the running practice on DNA methylation status of welltrained elderly subjects.

No significant differences were observed on DNA methylation levels between the SED and SED individuals at baseline. Conversely, our group recently reported that sedentary elderly individuals showed higher levels of global histone $\mathrm{H} 4$ acetylation when compared to the runners ones [25]. Taken together, these findings suggest that global histone $\mathrm{H} 4$ acetylation, and not DNA methylation status, may be consider an important epigenetic mark linked to endurance phenotype in well-trained-elderly individuals.

The current study also demonstrated that global DNA methylation plasma levels remained unaltered in the RUN group after the running race compared to the before period, evidencing that this epigenetic marker is not acutely altered in response to running in this population. Our data corroborate those obtained by Robson-Ansley and colleagues [26] reporting that a single bout of a prolonged and strenuous exercise protocol (running in a treadmill) was not able to alter DNA methylation levels in peripheral blood of well-trained young-aged men. In addition, global histone $\mathrm{H} 4$ acetylation levels also are not altered in response to a street running race in both middle-aged and elderly well-trained individuals $[25,10]$. Taken together, these results led us to hypothesize a possible epigenome adaptation in response to chronic exercise exposure in both young and aged-well trained individuals.

In contrast, a remarkable reduction on DNMT3B concentrations, an indicative of DNA hypomethylation status, was observed in peripheral blood of active young-aged males following a 120-min of treadmill running [6]. In addition, Zhang and colleagues showed a significant increase on DNA methylation levels in elderly sedentary individuals submitted to a walking interval training protocol during six months when compared to control ones [8]. Significant changes on global DNA methylation levels was also demonstrated in preciously sedentary COPD individuals submitted to a single exercise session [27]. Furthermore, sperm DNA was also demethylated in response a 3 months of exercise training in healthy young men. When combining these findings, it appears entirely suppose that the physical conditioning (sedentary versus trained) are a pivotal factor related to exercise modulation on DNA methylation levels. Specifically, that this epigenetic mark is remarkably responsive to short and long-term exercise interventions in previously sedentary people.

Among the benefits provided by the endurance exercise, increased anti-inflammatory cytokines in parallel to lack of changes in pro inflammatory mediators, representing lower T1/ T2 cytokine ratio, are directly to the intensity of the exercise [28]. The results of our study demonstrated that an acute bout of outdoor $5-10 \mathrm{~km}$ endurance challenge induced an increase in T2 inflammation, as demonstrated by higher IL-4 levels after the workout. These effects are attributed, at least in part, to the elevations of miokine levels, glycoproteins like cytokines that are express by the muscle tissue during and after physical stress [29].

Muscle contraction stimulates the secretion of IL- 6 with a variety of functions, especially the modulation of metabolism and the induction of an anti-inflammatory environment through the stimulation of anti-inflammatory cytokines [30]. Our data demonstrated a significant increase in systemic IL-6 immediately after bout in elderly runners. Despite the fact that IL-6 is recognized as a pro-inflammatory cytokine, acute exercise can induce an increase in IL-6 secretion from muscle cells with anti-inflammatory effects such as reduction of nucler-kappa beta factor expression in mononuclear cells, a master regulator of pro inflammatory transcription of DNA, and stimulation of T2 cytokines [29]. Marathons and ultra-endurance exercise can induce an elevation in order to 100x of IL-6 levels in serum of athletes after the bout [31,32].The increases in serum concentrations of IL- 6 observed in our study was more attenuated, however, we cannot rule out that these differences in IL- 6 after the running may be related to the duration of the race.

There has been consistent evidence that acute high intensity endurance exercise induce an elevation in IL-4 levels in both young trained or untrained individuals. In addition, cytokines regarding T1 inflammation, like INF- $\gamma$, are suppressed many hours after bout $[19,33]$. The suppression may last for more than 24 hours after bout [34]. Moreover, the changes in the cytokines produced by $\mathrm{T} 1$ and $\mathrm{T} 2$ cells are similar to falls observed in the frequency of the T cells that produce these cytokines [33]. Across qualitative analysis, Kakanis and coworkers [35] demonstrated an increase in IL-4 levels in addition to a T1/T2 shift after $2 \mathrm{~h}$ of cycling exercise at $90 \%$ of second ventilatory threshold. Interesting, a non-significant increase in T-cells expressing IL-4 in parallel to decreased the percentage of T cells expressing INF- $\gamma$ was observed after intensive treadmill running at 75\% of VO2Max $[20,21]$. Mechanistically, these changes in T1/T2 balance may be related to events associated to exercise-induced up-regulation of GATA3 and CCR4 genes, transcription factor genes related to IL-4 
and T2 phenotype [19]. In addition, higher expression of IL-6 was correlated with the percentage of CD8+ T cells that producing IL-4 after exercise [33].

Hormonal changes is also postulated as exerts effects on cytokine response. Glucorticoids (i.e. cortisol) binds in their cytoplasmic/nuclear receptors on antigen presenting cells to inhibits the expression of IL-12, a pivotal cytokine to induce T1 cells in adaptive immunity [36]. Catecholamines also suppress the production of INF- $\gamma$ and related T1 cytokines, and are able to stimulate the production of T2 cytokines [37]. It was demonstrated a negative correlation between adrenaline and the peripheral frequency of Th1 cells after $2 \mathrm{~h}$ of $75 \% \mathrm{VO} 2 \mathrm{max}$ endurance exercise [33]. In addition, T1 cells express higher density of $\beta$-adrenergic receptors compared with T2 cells. Thus, the higher cathecolaminergic influence of response can induce both trafficking of circulating T1 cells from the systemic circulation to inflamed tissue, which can reduce the frequency of T1 cells in peripheral circulation [38]. Taken together, these data may indicate a possible regulatory effect provided by the endurance exercise in the elderly group because IL- 4 and IL- 6 are synergic cytokines directing a T2-prone response [39].

\section{Conclusions}

In summary, our data presents a first attempt to bridge the gap between exercise and the modulation of DNA methylation status in well-trained elderly individuals. The findings here obtained could suggest that this epigenetic signal might not be consider a potential biomarker related to the elderly runnings phenotype as well exerts a stable profile in response to longterm running practice. Furthermore, the inflammatory profile presented by elderly runners in response to chronic and acute physical effort may indicate a possible regulatory effect provided by the endurance exercise on aging processes.

\section{Acknowledgements}

The authors would like to thank the following Brazilian agencies: Coordenação de Aperfeiçoamento de Pessoal de Nível Superior (CAPES), Conselho Nacional de Desenvolvimento Científico e Tecnológico (CNPq) and Fundação de Amparo à Pesquisa do Estado do Rio Grande do Sul (FAPERGS) for financial support.

\section{References}

1. World report on ageing and health WHO. 2015.

2. Elsner VR, Lovatel GA, Moyses F, Bertoldi K, Spindler C, Cechinel $L R$, et al. Exercise induces age-dependent changes on epigenetic parameters in rat hippocampus: a preliminary study. Exp Gerontol. 2013;48(2):136-139. Doi: 10.1016/j.exger.2012.11.011

3. Lovatel GA, Elsner VR, Bertoldi K, Vanzella C, Moyses Fdos S, Vizuete A, et al. Treadmill exercise induces age-related changes in aversive memory, neuroinflammatory and epigenetic processes in the rat hippocampus. Neurobiol Learn Mem. 2013;101:94-102.

4. Jones PA, Baylin SB. The fundamental role of epigenetic events in cancer. Nat Rev Genet. 2002;3(6):415-428.

5. Reik W, Kelsey G, Walter J. Dissecting de novo methylation. Nat. Genet. 1999:23(4);380-382.
6. Kouzarides T. Chromatin modifications and their function. Cell. 2007;128(4):693-705.

7. Garatachea N, Pareja-Galeano H, Sanchis-Gomar F, Santos-Lozano A, Fuiza-Luces C, Morán M, et al. Exercise Attenuates the Major Hallmarks of Aging. Rejuvenation Res. 2015;18 (1):57-89. Doi: 10.1089/ rej.2014.1623

8. Zhang Y, Hashimoto S, Fujii C, Hida S, Ito K, Matsumura T, et al. NFkB2 Gene as a Novel Candidate that Epigenetically Responds to Interval Walking Training. Int J Sports Med. 2015;36(9):769-775. Doi: 10.1055/s-0035-1547221

9. Knebel F, Schimke I, Schroeckh S, Peters H, Eddicks S, Schattke S, et al. Myocardial function in older male amateur marathon runners: assessment by tissue Doppler echocardiography, speckle tracking, and cardiac biomarkers. J Am Soc Echocardiogr. 2009;22(7):803-809. Doi: 10.1016/j.echo.2009.04.009

10. da Silveira FP, Basso C, Raupp W, Dalpiaz M, Bertoldi K, Siqueira IR, et al. BDNF levels are increased in peripheral blood of middle-aged amateur runners with no changes on histone $\mathrm{H} 4$ acetylation levels. J Physiol Sci. 2017;67(6):681-687. Doi: 10.1007/s12576-016-0496-6

11. Lavratti C, Dorneles G, Pochmann D, Peres A, Bard A, de Lima Schipper $\mathrm{L}$, et al. Exercise-induced modulation of histone $\mathrm{H} 4$ acetylation status and cytokines levels in patients with schizophrenia. Physiol Behav. 2017;168(1):84-90. Doi: 10.1016/j.physbeh.2016

12. Dorneles GP, da Silva IRV, Korb A, Bertoldi K, Siqueira IR, Elsner VR, et al. High Intensity interval exercise enhances the global HDAC activity in PBMC and anti-inflammatory cytokines of overweigthobese subjectis. Obesity Medicine 2016;(2):25-30. Doi: 10.1016/j. obmed.2016.05.004

13. Francechi $\mathrm{C}$ and Campisi J. Chronic Inflammation (Inflammaging) and Its Potential Contribution to Age-Associated Diseases. J Gerontol A Biol Sci Med Sci. 2014;69 (1): S4-S9.

14. Frasca D and Blomberg BB. Inflammaging decreases adaptive and innate immune responses in mice and humans. Biogerontology. 2016;17(1):7-19. Doi: 10.1007/s10522-015-9578-8

15. Mosmann TR, Cherwinski H, Bond MW, Giedlin MA, Coffman RL. Two types of murine helper T cell clone. I. Definition according to profiles of lymphokine activities and secreted proteins. J Immunol. 1986;136(7):2348-2357.

16.Seder RA, and Paul WE. Acquisition of lymphokine-producing phenotype by CD4+ T cells. Annu Rev Immunol 1994;12:635-673.

17. Jäger A, and Kuchroo VK. Effector and regulatory T-cell subsets in autoimmunity and tissue inflammation. Scand J Immunol. 2010;72(3): 173-184. Doi: 10.1111/j.1365-3083.2010.02432.x

18. Libetta C, Esposito P, Sepe V, Guastoni C, Zucchi M, Meloni F, and Dal Canton A. Effects of different peritoneal dialysis fluids on the TH1/ TH2 balance. Eur Cytokine Netw. 2011;22(1):24-31.

19. Xiang L, Rehn KE, Marshal GD. Effects of strenuous exercise on Th1/ Th2 gene expression from human peripheral blood mononuclear cells of marathon participants. Mol Immunol. 2014;2(60):129-134. Doi: 10.1016/j.molimm.2014.03.004

20. Ibfel T, Petersen EW, Bruunsqaard H, Sandmand M, Pedersen BK. Exercise-induced change in type 1 cytokine-producing CD8+ T cells is related to a decrease in memory T cells. J Appl Physiol. 2002;93(2):645648.

21. Mikkelsen UR, Cooupè C, Karlsen A, Grosset JF, Schjeerling, P, Mackey $\mathrm{AL}$, et al. Life-long endurance exercise in humans: Circulating levels 
of inflammatory markers and leg muscle size. Mech Ageing Dev. 2013;134(11-12):531-540. Doi: 10.1016/j.mad.2013.11.004

22. Kullo IJ, Khaleghi M, Hensrud DD. Markers of inflammation are inversely associated with VO2 max in asymptomatic men. J Appl Physiol. 2007;102(4):1374-1379.

23. Jankord R, Bozena J. Influence of Physical Activity on Serum IL-6 and IL-10 Levels in Healthy Older Men. Med Sci Sports Exerc. 2004;36(6):960-964.

24. Calvo DG, Garcia BF, Delgado BL, Gonzáles SR, Macia MG, Suárez FM, et al. Long-term training induces a healthy inflammatory and endocrine emergent biomarker profile in elderly men. Age 2012;34(3):761-771. Doi: $10.1007 / \mathrm{s} 11357-011-9266-9$

25. Figueiredo, Ineu A, Jose Cunha J, Reichert Vital da Silva I, Luna Martins L, Bard A, et al. Running-induced Functional Mobility Improvement in the Elderly Males is Driven by Enhanced Plasma BDNF Levels and the Modulation of Global Histone H4 Acetylation Status, Middle East J Rehabil Health Stud. 2017;4(3):e57486. doi: 10.5812/mejrh.57486

26. Robson-Ansley PJ, Saini A, Toms C, Ansley L, Walshe IH, Nimmo MA et al. Dynamic changes in DNA methylation status in peripheral blood mononuclear cells following an acute bout of exercise: potential impact of exerciseinduced elevations in interleukin- 6 concentration. J Biol Regul Homeost Agents. 2014;28(3):407-417.

27. da Silva IRV, de Araujo CLP, Dorneles GP, Peres A, Bard AL, Elsner VR, et .al. Exercise-modulated epigenetic markers and inflammatory response in COPD individuals: A pilot study. Respir Physiol Neurobiol. 2017;242:89-95. Doi: 10.1016/j.resp.2017.04.004

28. Zhao GZS, Davie A, Quansheng Su. Effects of moderate and high intensity exercise on T1/T2 balance. Exerc Immunol Rev. 2012;18:98114.

29. Benatti FB and Pedersen BK. Exercise as an anti-inflammatory therapy for rheumatic diseases-myokine regulation. Nat Rev Rheumatol. 2015;11(2):86-97. Doi: 10.1038/nrrheum.2014.193

30. Pal M, Febbraio MA, Whittham M. From cytokine to myokine: the emerging role of interleukin-6 in metabolic regulation. Immunol Cell Biol. 2014;92(4):331-339. Doi: 10.1038/icb.2014.16
31. Shin, YO and Lee JB. Leukocyte chemotactic cytokine and leukocyte subset responses during ultra-marathon running. Cytokine 2013;61(2):364-369. Doi: 10.1016/j.cyto.2012.11.019

32. Stelzer L, Kröpfl JM, Fuchs R, Pekovits K, Mangge H, Raggam RB, et al. Ultra-endurance exercise induces stress and inflammation and affects circulating hematopoietic progenitor cell function. Scan J Med Sci Sports. 2015;25(5):442-450. Doi: 10.1111/sms.12347

33. Steensberg A, Toft AD, Bruunsgaard H, Sandmand M, HalkjaerKristensen J, Pedersen BK. Strenuous exercise decreases the percentage of type 1 T cells in the circulation. J appl Physiol. 2001;91(4):17081712. Doi: 10.1152/jappl.2001.91.4.1708

34. Baum M, Müller-Steinhardt M, Liesen H, Kirchner H. Moderate and exhaustive endurance exercise influences the interferon-gamma levels in whole-blood culture supernatants. Eur J Appl Physiol. 1997;76(2):165-169.

35. Kakanis MW, Peake J, Brenu EW, Simmonds M, Gray B, MarshalGraidsnik SM. T helper cell cytokine profiles after endurance exercise. J Interferon Cytokine Res. 2014;34(9): 699-706. Doi: 10.1089/ jir.2013.0031

36. Blotta MH, DeKruyff RH, Umetsu DT. Corticosteroids inhibit IL-12 production in human monocytes and enhance their capacity to induce IL-4 synthesis in CD4+ lymphocytes. J Immunol. 1997;158(12):55895595.

37. Smith LL. Overtraining, excessive exercise, and altered immunity: is this a T helper- 1 versus T helper- 2 lymphocyte response? Sports Med. 2003;33(5):347-364.

38. Lancaster GI, Halson SL, Khan Q, Drysdale P, Wallace F, Jeukendrup AE, et al. Effects of acute exhaustive exercise and chronic exercise training on type 1 and type 2 T lymphocytes. Exerc Immunol Rev 2004;10:91106.

39. Sokol CL, Barton GM, Farr AG, Medzhitov R. A mechanism for the initiation of allergen-induced $\mathrm{T}$ helper type 2 responses. Nat Immunol. 2008;9(3):310-318. Doi: 10.1038/ni1558 